VOL. 60 (1999) [403-405]

\title{
A NOTE ON THE $2 k$-TH MEAN VALUE OF THE HURWITZ ZETA FUNCTION
}

\section{A. KUMCHeV}

Consider the error term in the asymptotic formula

$$
\int_{0}^{1}\left|\zeta_{1}(1+i t, \alpha)\right|^{2 k} d \alpha=A(k)+O\left(|t|^{-\delta(k)} \log |t|\right) .
$$

In this note we obtain $\delta(k) \asymp 1 /\left(k^{6} \log ^{2} k\right)$ which, for large values of $k$, presents a substantial improvement over the previously known result $\delta(k) \approx 1 /\left(k^{2} 2^{k^{2}}\right)$.

For complex $s=\sigma+i t$ and real $\alpha, 0<\alpha<1$, the Hurwitz zeta function is defined by

$$
\zeta(s, \alpha)=\sum_{n=0}^{\infty} \frac{1}{(n+\alpha)^{s}},
$$

if $\sigma>1$, and then continued analytically on $\mathbb{C} \backslash\{1\}$ via a functional equation similar to the one for the Riemann zeta function [2, Sections 1.2 and 1.4]. Let

$$
\zeta_{1}(s, \alpha)=\zeta(s, \alpha)-\alpha^{-s} .
$$

Recently, Wang [3] proved the asymptotic formula

$$
\int_{0}^{1}\left|\zeta_{1}(1+i t, \alpha)\right|^{2 k} d \alpha=A(k)+O\left(|t|^{-\delta(k)} \log |t|\right)
$$

where $A(k)$ and $\delta(k)>0$ are explicit constants depending only on $k$. In this note we are concerned with the error term in (1) for large values of $k$. Via van der Corput's estimates for the arising zeta sums, in [3], $\delta(k)$ of order $1 /\left(k^{2} 2^{k^{2}}\right)$ was shown to be admissible in (1). Applying Vinogradov's method (which is the natural approach in this situation), we show that one can take $\delta(k) \asymp 1 /\left(k^{6} \log ^{2} k\right)$. We establish the following

THEOREM. There exists an absolute constant $c>0$ such that for any real $t,|t|>t_{0}$, and for any positive integer $k$, the asymptotic formula (1) holds with

$$
\delta(k)=\frac{c}{k^{6} \log ^{2}(2 k)} .
$$

Received 9th February, 1999

Copyright Clearance Centre, Inc. Serial-fee code: 0004-9727/99 \$A2.00+0.00. 
The constant $A(k)$ and the implied constant depend only on $k$.

As one would expect, the explicit value for $c$, although effectively computable, is too small to justify its place in the statement of the Theorem. For $k \geqslant 3$, certainly $c=2^{-130}$ will suffice, and this value of $c$ would improve over the result in [3] for such $k$. For large $k$ a better value of $c$ (say $10^{-7}$ ) can easily be obtained.

PROOF OF THE THEOREM: Since the argument is similar to that in [3], we give just a brief sketch. Let

$$
\theta(k)=\frac{1}{4 k+2}\left(\frac{1}{2 k+1}-\delta(k)\right)
$$

and set $N=|t|^{\theta(k)}$. By $[3,(3.4)$ and $(3.5)]$,

$$
\int_{0}^{1}\left|\sum_{n=1}^{N} \frac{1}{(n+\alpha)^{1+i t}}\right|^{2 k} d \alpha=A(k)+O\left(N^{-1}\right)+O(|S|)
$$

with $A(k)=\int_{0}^{1} \zeta_{1}(2, \alpha)^{k} d \alpha$ and

$$
S=\sum_{n_{1}, \ldots, n_{2 k}=1}^{N} \int_{0}^{1} \frac{\left(n_{1}+\alpha\right)^{-i t} \cdots\left(n_{2 k}+\alpha\right)^{i t}}{\left(n_{1}+\alpha\right) \cdots\left(n_{2 k}+\alpha\right)} d \alpha
$$

where the sum is only over $2 k$-tuples in which $n_{1}, \ldots, n_{k}$ do not form a permutation of $n_{k+1}, \ldots, n_{2 k}$. Sharpening the last inequality in the proof of $[3$, Lemma 2], we find that each specific term in $S$ is

$$
\ll \frac{\left(|t|^{-1} N^{8 k(k+1)}\right)^{1 /(2 k+1)}}{n_{1} \cdots n_{2 k}}
$$

and, hence,

$$
\int_{0}^{1}\left|\sum_{n=1}^{N} \frac{1}{(n+\alpha)^{1+i t}}\right|^{2 k} d \alpha=A(k)+O\left(N^{-1}\right)+O\left(|t|^{-1 /(2 k+1)} N^{4 k+2}\right) .
$$

Thus, it suffices to show that

$$
\zeta_{1}(1+i t, \alpha)=\sum_{n=1}^{N} \frac{1}{(n+\alpha)^{1+i t}}+O\left(|t|^{-\delta(k)}\right) .
$$

This approximate formula follows from the approximate functional equation for $\zeta_{1}(s, \alpha)$ [2, Theorem III.2.1] and the estimate

$$
\left|\sum_{x<n \leqslant 2 x}(n+\alpha)^{-i t}\right| \ll x|t|^{-\delta(k)} \quad(N<x \leqslant|t|) .
$$

If $N \leqslant x \leqslant|t|^{1 / 121}$, (2) can be derived from [1, Theorem III.1.3] via Vinogradov's method (see for example [2, Theorem IV.2.1]); if $|t|^{1 / 121} \leqslant x \leqslant|t|$, one can use van der Corput's method of exponent pairs. This completes the proof. 


\section{REFERENCES}

[1] G.I. Arkhipov, V.N. Chubarikov and A.A. Karatsuba, Theory of multiple exponential sums, (in Russian) (Nauka, Moscow, 1987).

[2] A.A. Karatsuba and S.M. Voronin, The Riemann zeta function (Walter de Gruyter \& Co., Berlin, 1992).

[3] Y. Wang, 'On the $2 k$-th mean value of Hurwitz zeta function', Acto Math. Hungar. 74 (1997), 301-307.

Department of Mathematics

University of South Carolina

Columbia SC 29208

United States of America

e-mail: koumtche@math.sc.edu 